\title{
The effects of epidural versus intravenous patient-controlled analgesia on postoperative outcomes in elderly patients who have undergone gastrectomy: a retrospective trial
}

\section{Yun-kwang Kim, Ji Heui Lee, Seok Hee Kang, Yongjoon Choi, Ji-yeon Lee, So Young Lee, Su-Nam Lee, Eun-Ju Lee, and Cheong Lee}

Revised 1st, April 29, 2017

2nd, June 12, 2017

Accepted June 20, 2017

\section{Corresponding author}

Ji Heui Lee, M.D., Ph.D.

Department of Anesthesiology and Pain Medicine, Korea Cancer Center Hospital, 75, Nowon-ro, Nowon-gu, Seoul 01812, Korea

Tel: 82-2-970-2820

Fax: 82-2-970-2413

E-mail: jiheui0255@naver.com
Department of Anesthesiology and Pain Medicine, Korea Cancer Center Hospital, Seoul, Korea

Background: Efforts for improving surgical outcomes in elderly patients should include the prevention of perioperative complications and proper postoperative pain management. Epidural analgesia is effective in decreasing perioperative stress and postoperative complications, and in improving recovery. Recently, it has been suggested that epidural analgesia may attenuate immune suppression during the perioperative period and lead to reductions in cancer recurrence and improvements in overall survival. Assuming that these effects of epidural analgesia are present in vulnerable elderly patients, we compared the efficacy of epidural patient-controlled analgesia and intravenous patientcontrolled analgesia on postoperative outcomes in elderly patients who had undergone gastrectomy.

Methods: We evaluated 214 elderly patients who had undergone elective open gastrectomy for gastric cancer from 2011 to 2014 . The demographic characteristics, analgesic efficacy, complications within the first 30 postoperative days, hospital stay, 24-month postoperative morbidity, cancer recurrence, and mortality were studied retrospectively.

Results: Two-hundred and five patients were analyzed. Eighty-five patients received epidural patient-controlled analgesia (EPCA group) and 120 patients received intravenous patient- controlled analgesia (IVPCA group). The maximum pain scores were decreased to a greater extent in the EPCA group on the day of the operation $(P<0.001)$ and on the first postoperative day $(P=0.001)$ when compared to the IVPCA group. The patients in the EPCA group also required less analgesics on the day of the operation $(P=0.033)$ than those in the IVPCA group. The effect of EPCA on complications within the first 30 postoperative days, 24-month postoperative morbidity, cancer recurrence, and mortality were negligible in our study.

Conclusions: EPCA provided more effective pain control than IVPCA in elderly patients who had undergone elective gastric cancer surgery, but did not affect postoperative outcomes.

Key Words: Elderly, Epidural analgesia, Injections, Intravenous, Neoplasm recurrence, Outcomes.

This is an Open Access article distributed under the terms of the Creative Commons Attribution Non-Commercial License (http://creativecommons.org/licenses/by-nc/4.0) which permits unrestricted non-commercial use, distribution, and reproduction in any medium, provided the original work is properly cited. 


\section{INTRODUCTION}

As the global population ages and geriatric patients increase in number, anesthesiologists will be asked to care for a greater number of aged patients. The aging process induces anatomical, functional, and physiological changes in the major organs of the body. These changes may significantly affect postoperative outcomes. A previous study revealed that postoperative morbidity and mortality increased in geriatric patients when compared to younger patients [1].

Neuraxial anesthesia and analgesia is effective in decreasing postoperative complications, alleviating surgical stress, and accelerating recovery $[2,3]$. Moreover, regional analgesic techniques have been suggested to attenuate host immune suppression during cancer surgery, and to be associated with reduced cancer recurrence or better overall survival after cancer surgery $[4,5]$. In fact, high-risk patients undergoing major surgery may especially benefit from postoperative epidural analgesia [6].

However, there is no data available regarding the effects of epidural analgesia on postoperative complications, mortality, or cancer-related outcomes in elderly patients, who are likely to have more morbidities. We hypothesized that epidural patient-controlled analgesia (EPCA) would have an analgesic effect superior to that of intravenous patient-controlled analgesia (IVPCA) in elderly patients. Additionally, we expected the positive effects of EPCA to improve postoperative outcomes in elderly patients. To test this hypothesis, we performed the present study, which was designed as a retrospective clinical trial in elderly patients who had undergone gastrectomy.

\section{MATERIALS AND METHODS}

After obtaining approval from our Institutional Review Board, we registered this study at http://clinicaltrials.gov (registration number: KCT0002153). We reviewed the electronic medical records of patients aged 65 years or older with American Society of Anesthesiologists physical status classifications of II to III who had undergone elective open gastrectomy for stomach cancer between May 2011 and May 2014. The exclusion criteria were as follows: emergent operation; recurrent or metastatic gastric cancer; preoperative deficits in cognition or consciousness; and preoperative neurologic or psychotic diseases, such as seizure, schizophrenia, and Parkinson's disease. All patients received bowel preparation on the day before the surgery and were subjected to the same standard anesthetic technique. Informed consent regarding the benefits and risks of EPCA and IVPCA was received from the participants. The analgesic method was chosen based on the preferences of the patients, surgeons, and anesthesiologists.

Anesthesia was induced using thiopental, lidocaine, rocuronium, or vecuronium. Anesthesia was maintained with $0.5-2$ vol\% of sevoflurane in a $50 \%$ oxygen and $50 \%$ nitrous oxide mixture. Repeated boluses of rocuronium or vecuronium were administered for muscle relaxation during the surgery, as necessary. The following parameters were monitored in all patients: electrocardiography, noninvasive or invasive blood pressure, oximetry, urine output, capnography, and bispectral index. A nasogastric tube was inserted after intubation.

After the induction of general anesthesia, epidural catheterization was performed by a faculty anesthesiologist highly experienced in this procedure in the EPCA group. The epidural catheter was inserted at the T8-10 level, depending on the anatomy of patients and the preference of the anesthesiologist. Subarachnoid or intravascular placement of the epidural catheter was ruled out based on the absence of blood or cerebrospinal fluid aspiration. Both analgesic management procedures began at the time of peritoneal closure. An epidural bolus dose (30-50 $\mu$ g of fentanyl and $5 \mathrm{ml}$ of $0.15 \%$ bupivacaine) and intravenous $75 \mu \mathrm{g}$ of palonosetron were administered in the EPCA group. Subsequently, an EPCA pump (Automed 3200; Ace Medical Co., Ltd., Korea) containing a mixture (total volume, $100 \mathrm{ml}$ ) of $0.15 \%$ bupivacaine and fentanyl was connected to the catheter. The basal infusion rate of the pump was $1 \mathrm{ml} / \mathrm{h}$, with 4 -ml bolus doses allowed every 30 minutes. In the IVPCA group, the patients received a basal infusion of $1 \mathrm{ml} / \mathrm{h}$ with boluses of $1 \mathrm{ml}$ and a lockout interval of 15 minutes using a pump (Autoselector; Ace Medical Co., Ltd., Korea) containing $100 \mathrm{ml}$ of a solution containing fentanyl (with or without ketorolac). The doses of fentanyl delivered via the epidural or intravenous route and the addition of adjunctive analgesics, such as ketorolac for IVPCA, were at the discretion of each anesthesiologist. Additional administration of analgesics (fentanyl, meperidine, or ketorolac) was allowed for breakthrough pain on an as-needed basis in both groups. Antiemetics (palonosetron or ramosetron) were 
used if a patient experienced nausea or vomiting. If severe sedation was observed, the infusion of PCA was stopped until the patient became alert. The epidural catheter or IVPCA was removed after the delivery of the analgesics.

The following data were collected from the patients' electronic medical records: age, sex, body mass index (BMI), American Society of Anesthesiology (ASA) physical status class, type of surgery and surgeon, multivisceral resection, length of surgery, comorbidities, tumor/lymph node/metastasis (TNM) stage, and postoperative pain score. We also investigated the incidence of complications within the first 30 postoperative days (POD), as well as hospital stay, morbidity, mortality, and cancer recurrence from 2 to 24 months after the gastrectomy. Multivisceral resection was defined as any additional organ resection, excluding that of the stomach or an adjacent lymph node. Numerical rating scores for postoperative pain (NRS: 0-10) for each patient were obtained from electronic records, as documented in the nursing assessment forms. The highest recorded scores and the numbers of supplemental analgesia requests were noted for the day of the operation and on the first POD. In most cases, EPCA and IVPCA were discontinued between the second and fourth POD. Patients who had their PCA removed before the second POD were considered to have an ineffective PCA and were excluded from the analysis.

Postoperative complications were ascertained by a retrospective review of medical records by every surgeon and nurse. Pneumonia was defined as a condition with an infiltrate on chest radiograph and clinical findings such as fever, leukocytosis, purulent secretions, or increasing oxygen requirements necessitating the administration of antibiotics according to the 2004 guidelines of the American Thoracic Society [7]. Prolonged postoperative ileus (PPOI) was defined as a situation wherein two or more of the following was present: nausea, inability to tolerate oral diet over 24 hours, absence of flatus over 24 hours, abdominal distension, and radiological confirmation on or after the fourth POD without prior resolution of postoperative ileus (POI) [8]. Delirium was assessed using medical records. Evidence of delirium included confusion, agitation, sedation, hallucinations and delusions, or diagnosis by a psychologist. Recurrence-free survival was defined as the period from the day of the operation to the day on which the first recurrence was diagnosed or the last followup during the 24-month period. Overall survival was defined as the period from the day of the operation until death or the last follow-up during the 24-month period.

\section{Statistical analysis}

The sample size was fixed based on the number of available patients during a given period because our study was naturally retrospective. Clinical data are described as mean and standard deviation for continuous variables (age, BMI, surgical time, NRS, used dose of fentanyl), median (interquartile range) for discrete variables (analgesic requirement), and number (percentage) for categorical variables (sex, ASA, coexisting disease, surgical type, multivisceral resection, staging, transfusion, chemotherapy, and various postoperative complications). The Kolmogorov-Smirnov test was used to test continuous variables for normality. Parametric variables were analyzed using Student's $t$ tests. Discrete variables and categorical variables were compared using Mann-Whitney $U$ tests and chi-square tests, respectively. Survival curves were analyzed using the Kaplan-Meier method and were compared using the log-rank test. Statistical analyses were performed using SPSS statistical package (Windows version 23.0; SPSS Inc., USA). Statistical significance was determined using $P$ values $<0.05$.

\section{RESULTS}

Two-hundred and fourteen patients were assessed in the study. Nine patients were excluded from the statistical analysis due to failure of EPCA or IVPCA. The reasons for early EPCA discontinuation (3 cases) were nausea, hypotension accompanied by severe sedation, and inadvertent catheter removal. IVPCA failures occurred in 6 cases due to nausea ( 3 cases), postoperative bleeding, mechanical error, and dizziness. The study therefore ultimately included 205 patients, with 120 patients in the IVPCA group and 85 patients in the EPCA group.

The demographics of the patients are shown in Table 1. There were no differences in coexisting diseases, including diabetes mellitus, hypertension, pulmonary disease, cardiovascular disease, or central nervous system disease between the groups. No significant differences were observed in surgical time, surgical type, multivisceral resection, TNM classification, or staging between the groups, although a slightly 
Table 1. Demographic Data

\begin{tabular}{|c|c|c|c|}
\hline & $\operatorname{EPCA}(n=85)$ & $\operatorname{IVPCA}(n=120)$ & $P$ value \\
\hline \multicolumn{4}{|l|}{ Patient characteristics } \\
\hline Age & $73.0 \pm 4.9$ & $72.4 \pm 4.5$ & 0.266 \\
\hline Male/female & $47 / 38$ & $79 / 41$ & 0.084 \\
\hline $\mathrm{BMI}$ & $23.5 \pm 3.2$ & $23.4 \pm 3.1$ & 0.134 \\
\hline \multicolumn{4}{|l|}{ ASA physical status } \\
\hline II/III & $52 / 33$ & $77 / 43$ & 0.662 \\
\hline \multicolumn{4}{|l|}{ Comorbidities } \\
\hline $\mathrm{DM}$ & $26(31)$ & $31(26)$ & 0.454 \\
\hline HT & $43(51)$ & $55(46)$ & 0.502 \\
\hline Pulmonary disease & $11(13)$ & $21(8)$ & 0.376 \\
\hline$C-V$ & $8(9)$ & $11(9)$ & 0.952 \\
\hline CNS & $4(5)$ & $15(13)$ & 0.060 \\
\hline Others & $14(17)$ & $22(18)$ & 0.730 \\
\hline \multicolumn{4}{|l|}{ Number of diseases } \\
\hline $0 / 1 / 2 / 3 / 4 / 5$ & $18 / 38 / 16 / 11 / 2 / 0$ & $23 / 54 / 32 / 7 / 3 / 1$ & 0.418 \\
\hline \multicolumn{4}{|c|}{ Surgical and oncologic data } \\
\hline Surgical time (min) & $177 \pm 52$ & $167 \pm 45$ & 0.484 \\
\hline Surgical type & & & 0.907 \\
\hline STG, B I & $54(64)$ & $74(62)$ & \\
\hline STG, B II & $7(8)$ & $12(10)$ & \\
\hline $\mathrm{TG}$ & $24(28)$ & $34(28)$ & \\
\hline \multicolumn{4}{|c|}{ Multivisceral resection } \\
\hline No/Yes & $71 / 14(84 / 16)$ & $98 / 22(82 / 18)$ & 0.427 \\
\hline \multicolumn{4}{|l|}{ Staging } \\
\hline I/II/III & $39 / 29 / 17$ & $60 / 38 / 22$ & 0.844 \\
\hline \multicolumn{4}{|l|}{ TNM classification } \\
\hline $\mathrm{T} 1 / \mathrm{T} 2 / \mathrm{T} 3 / \mathrm{T} 4$ & $31 / 25 / 20 / 9$ & $50 / 41 / 23 / 6$ & 0.353 \\
\hline NO/N1/N2/N3 & $49 / 18 / 9 / 9$ & $66 / 27 / 10 / 17$ & 0.831 \\
\hline \multicolumn{4}{|c|}{ Perioperative transfusion } \\
\hline No/Yes & $73 / 12(86 / 14)$ & $110 / 10(92 / 8)$ & 0.187 \\
\hline \multicolumn{4}{|c|}{ Postoperative chemotherapy } \\
\hline No/Yes & $33 / 52(39 / 61) *$ & $64 / 56(53 / 47)$ & 0.040 \\
\hline
\end{tabular}

Data are shown as mean \pm SD or number of patients (\%). BMl: body mass index, ASA: American Society of Anesthesiologists, DM: diabetes mellitus, HT: hypertension, C-V: cardiovascular disease, CNS: central Nervous System, STG: subtotal gastrectomy, B: Billroth, TG: total gastrectomy, TNM: tumor/lymph node/metastasis. *P $<0.05$ when compared to IVPCA.

Table 2. Postoperative Analgesic Outcomes

\begin{tabular}{lccr}
\hline & EPCA $(n=85)$ & IVPCA $(n=120)$ & P value \\
\hline Maximum score of NRS & & & \\
POD 0 & $4.6 \pm 2.3^{*}$ & $6.9 \pm 1.6$ & 0.001 \\
POD 1 & $5.1 \pm 2.4^{*}$ & $6.2 \pm 2.1$ & 0.001 \\
The number of supplemental analgesia requests & & & $<0.001$ \\
POD 0 & $0[0-1]^{*}$ & $2[1-3]$ & 0.074 \\
POD 1 & $1[0-2]$ & $1[0-2]$ & 0.079 \\
Used dose $(\mu \mathrm{g})$ of fentanyl in PCA & $1,470 \pm 226$ & $1,480 \pm 184$ & \\
Uses of ketorolac in PCA & & & $<0.001$ \\
No/Yes & $85 / 0(100)^{*}$ & $86 / 34(72 / 28)$ & \\
\hline
\end{tabular}

Data are shown as mean $\pm \mathrm{SD}$, median [interquartile range], or number of patients (\%). NRS: numeric rating score of pain, POD: postoperative day, PCA: patient-controlled analgesia. * $\mathrm{P}<0.05$ when compared to IVPCA. 
higher number of patients underwent postoperative chemotherapy in the EPCA group $(\mathrm{P}<0.05)$.

Compared to the IVPCA group, maximum pain scores were significantly lower in the EPCA group until POD $1(\mathrm{P}<0.001)$ (Table 2). There were fewer requests for supplemental analgesics on the day of the operation in the EPCA group than in the IVPCA group $(\mathrm{P}<0.001)$. There were no differences in the dose of fentanyl used between the 2 groups. In the IVPCA group, $28.3 \%$ of the patients were co-administered ketorolac.

Postoperative cardiovascular complications, cerebrovascular attacks, wound problems, PPOI, pneumonia, bile leakage, hemorrhage, and delirium were comparable between the two groups (Table 3). No complications related to epidural analgesia were reported during the study period. Twentyfour-month morbidities in the EPCA group included neurologic disease ( 1 case), cancer ( 1 case), surgery-related disease (6 cases), and pulmonary disease (2 cases). Twenty-fourmonth morbidities in the IVPCA group included pulmonary disease ( 3 cases), renal disease ( 2 cases), surgery-related disease (3 cases), and neurologic disease (3 cases). There were no significant differences in the length of hospital stay or the 24-month morbidity rate between the two groups. Recurrence-free survival and overall survival were also similar between the two groups (Figs. 1 and 2). The causes of death were cancer ( 1 case) and pneumonia ( 2 cases) in the EPCA group, and cancer (3 cases), suicide (1 case), and pneumonia (1 cases) in the IVPCA group.

Table 3. Postoperative Complications and Outcomes Following Gastrectomy

\begin{tabular}{lccc}
\hline & $\begin{array}{c}\text { EPCA } \\
(\mathrm{n}=85)\end{array}$ & $\begin{array}{c}\text { IVPCA } \\
(\mathrm{n}=120)\end{array}$ & P value \\
\hline Complications within the first & 30 PODs, $\mathrm{n}(\%)$ & \\
C-V & $1(1.2)$ & $3(2.5)$ & 0.449 \\
CVA & $1(1.2)$ & $1(0.8)$ & 0.346 \\
Wound problem & $15(18)$ & $17(14)$ & 0.499 \\
PPOI & $23(27)$ & $20(17)$ & 0.073 \\
Bile leakage & $0(0)$ & $3(2.5)$ & 0.198 \\
Pneumonia & $5(6)$ & $6(5)$ & 0.509 \\
Atelectasis & $16(15)$ & $19(16)$ & 0.575 \\
Delirium & $2(2.4)$ & $3(2.5)$ & 0.658 \\
Hospital stays (days) & $11 \pm 4$ & $12 \pm 5$ & 0.172 \\
24-month morbidity, $\mathrm{n}(\%)$ & $10(11)$ & $11(9.2)$ & 0.546 \\
\hline
\end{tabular}

Data are shown number of patients (\%). C-V: cardiovascular complication, PODs: postoperative days, CVA: cerebrovascular accident, PPOI: prolonged postoperative ileus.

\section{DISCUSSION}

Epidural analgesia is the preferred form of analgesia after major surgery and has been studied as a measure to improve postoperative outcomes. Rodgers et al. [3] reported that epidural analgesia reduces serious postoperative complications and mortality. When compared to patients who had undergone abdominal surgery without epidural analgesia, the rates of venous thromboembolism, ileus, pneumonia, and immunosuppression are lower in patients with epidural

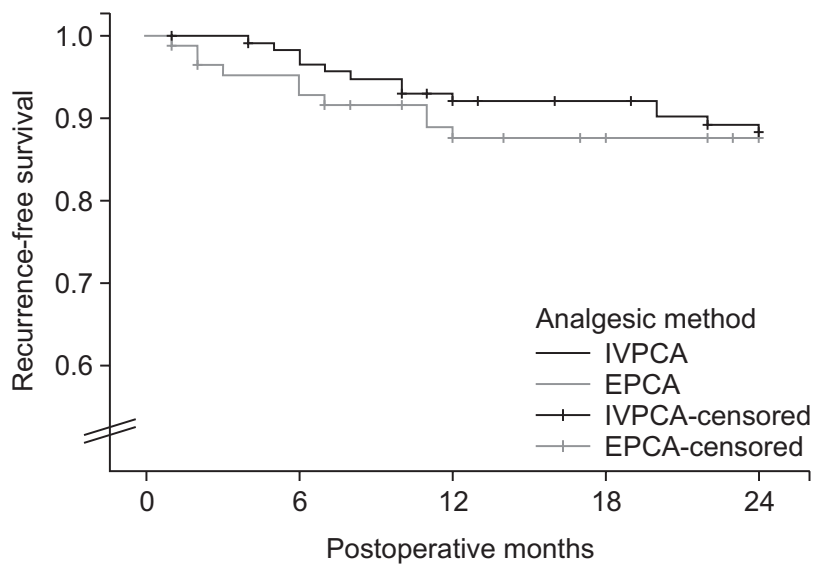

Fig. 1. Recurrence-free survival of 85 patients provided with epidural patient-controlled analgesia and 120 patients provided with intravenous patient-controlled analgesia. Kaplan-Meier estimate with 95\% confidence interval. Slash marks represent censored values $(P=0.743)$.

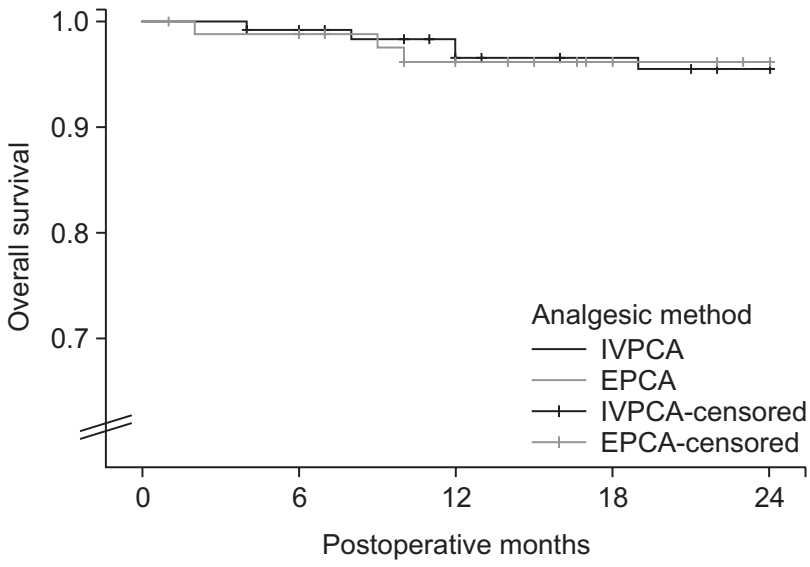

Fig. 2. Overall survival of 85 patients provided with epidural patient-controlled analgesia and 120 patients provided with intravenous patient-controlled analgesia. Kaplan-Meier estimate with 95\% confidence interval. Slash marks represent censored values $(P=0.872)$. 
analgesia [9]. A study [10] including a meta-analysis and a large randomized clinical trial showed that epidural analgesia leads to better analgesic efficacy and reduces the incidence of postoperative respiratory failure, especially in high-risk patients. Our study also revealed that EPCA had an analgesic effect superior to that of IVPCA in geriatric patients, who have increased perioperative risks after gastrectomy [11]. However, there were no differences between the two groups in the incidences of complications within the first 30 PODs, including cardiovascular complications, cerebrovascular accidents, wound problems, bile leakage, pulmonary complications, or delirium. The rates of cancer recurrence, morbidity, and mortality were comparable between the EPCA and IVPCA groups.

Epidural analgesia is supposedly associated with an earlier recovery of bowel function than systemic opioid-based analgesia [12]. There is no universally accepted clinical definition for POI, and PPOI is considered to be a more clinically problematic and pathologically significant entity [8]. In our study, there were no significant differences in the incidence of PPOI in the two groups.

Reports of the effects of epidural analgesia on postoperative outcomes have been controversial. Some authors have reported that they were unable to find sufficient evidence to conclude that epidural analgesia had relevant effects $[13,14]$. This may be attributable to improved adjunctive analgesics, such as nonsteroidal anti-inflammatory drugs, use of patientcontrolled analgesia, and reduction in overall surgical risk. Postoperative pain induces an endocrine-metabolic response, leads to nausea and ileus, stimulates an autonomic reflex, and increases muscular spasm. It is one of the most critical factors leading to increased perioperative stress. Elderly patients have an increased threshold for pain and may therefore complain of pain less frequently $[15,16]$. Therefore, in elderly patients, the beneficial effects of epidural analgesia may contribute less towards reducing postoperative complications and surgical outcomes.

Some studies $[17,18]$ have reported that epidural analgesia provides inadequate postoperative pain relief or has a lesser analgesic effect than IVPCA. Reasons for ineffective epidural analgesia may include suboptimal dosing of analgesics, incorrect placement of the catheter, or the migration of the catheter after correct placement [19]. Failure rates of $32 \%$ and $27 \%$ in thoracic and lumbar epidural catheterization, respectively, were reported in a cohort study of 2,140 patients [20]. It has recently been recognized that this procedure is not universally successful and that failure of adequate analgesia is experienced by $20-30 \%$ of patients [21]. Misplacement of epidural catheters in the paravertebral space, pleural cavity, or intravascular space has been reported. In this study, all epidural catheterizations were performed by a highly experienced anesthesiologist, who had been working as a pain consultant for decades. Therefore, there was an obvious analgesic effect in the EPCA group when compared to the IVPCA group.

Although generally safe, epidural catheterization is not entirely free of risks and complications. It is reported that the incidence of procedure-related complications, which are mostly minor and self-limiting, is approximately $7.8 \%$ [22]. The potential risk for these complications may be increased in elderly patients, who have a greater variety of vertebral deformations. In our study, we did not encounter any complications related to the epidural catheter.

In general, patients older than 65 years of age are defined as elderly due to functional loss of organs, delayed recovery from various stresses, and decreased socioeconomic function. Eighty percent of elderly surgical patients have at least 1 coexisting disease and one-third of them have 3 or more diseases. These various problems may thus increase the risk of perioperative morbidity and mortality [23]. Approximately $80 \%$ of the patients in our study also had 1 or more coexisting diseases. Even with the improvements in surgical and anesthetic techniques, postoperative complications and mortality after gastrectomy are significantly high in elderly patients [9]. When compared to another study [10] of morbidity after gastrectomy, we observed a lower incidence of postoperative morbidity and shorter surgical times and hospital stays. These factors may be associated with the negligible effects of epidural analgesia on postoperative outcomes.

Postoperative delirium is more common in older patients, and higher postoperative pain scores are related to postoperative delirium [24]. In this study, although patients in the EPCA group had lower postoperative pain scores, there was no difference in postoperative delirium between the two groups.

Recent studies have reported that regional anesthesia and analgesia might attenuate the proliferation of minimal residual disease after cancer surgery $[25,26]$. In a retrospective 
study of colectomies, there was no difference in cancer recurrence between patients treated with epidural analgesia vs. intravenous analgesia, although epidural analgesia was potentially beneficial in elderly patients [26]. Another study [27], however, reported that epidural analgesia was not associated with reduced recurrence of cancer, even in elderly patients. We also found that there were no differences in recurrencefree survival or overall survival between the epidural analgesia group and the intravenous analgesia group.

Vulnerability to postoperative complications in elderly patients may contribute to improper or insufficient postoperative pain therapy. Inadequate postsurgical pain management may be associated with poor interventional therapy, potentially higher incidence of medical complications, decreased analgesia for the patient, and patient and family member dissatisfaction. We did not administer bolus doses of analgesics for the prevention of early postoperative pain in the IVPCA group. This may have been associated with the higher pain score in the IVPCA group during the early postoperative period.

There is controversy regarding the site of action of fentanyl. There are also contradictory results regarding the effectiveness of epidural vs. intravenous fentanyl $[28,29]$. Epidural fentanyl has been suggested to have both systemic and spinal actions. It may therefore be more effective than intravenous fentanyl [29]. In our study, we used similar doses of fentanyl in the regimens for epidural and intravenous PCA. This may explain the reduced pain score in the EPCA group.

Previous studies have mainly focused on the effectiveness of the two different strategies for postoperative pain control. However, this trial is the first to compare the two methods with regard to their influence on postoperative morbidity and mortality and their effects on cancer recurrence in elderly patients after gastrectomy.

Our study had several major limitations, as it was a retrospective single-center trial. Patients were not randomly assigned to the IVPCA and EPCA groups in this retrospective trial. Therefore, there is a potential for bias, which may lead to imbalances in important measures, such as demographic data. For example, the incidence of postoperative chemotherapy was higher in the EPCA group than in the IVPCA group. Although we expected to find patients with more advanced cancer in the EPCA group, there was no evidence of an increased risk of cancer recurrence in this group. Therefore, we may be able to reach statistical significance between the groups with sufficient power to detect a small difference in cancer recurrence. Our study only included cases from a single institution and is thus inadequate for reaching universal conclusions. A multicenter randomized prospective study [27] compared major postoperative complications and mortality between patients who had been subjected to epidural vs. opioid analgesia. However, the population in the above study was very heterogeneous in terms of surgical teams, type of cancer, and clinical cancer protocols. In contrast, our study is a single-center study with no marked changes in surgical techniques or clinical cancer protocols. Finally, the short follow-up time may have affected cancer recurrence and overall survival. Follow-up and cut-off end-points have been variable in previous studies. Although 5-year recurrence-free survival and overall survival are usually reported in oncology studies, another study [25] has reported patient survival at less than 3 years. A study of colon cancer surgery observed patients for 10 years, but revealed that epidural supplementation was associated with enhanced survival only during the first 18 months [30]. There were difficulties in determining the adequate follow-up duration for cancer recurrence, especially in geriatric patients. We thus followed patients for only 2 years. Prospective and randomized studies with long follow-up periods may be necessary to determine the effects of analgesic techniques on cancer recurrence and overall survival. In conclusion, although epidural patient-controlled analgesia was more effective for postoperative pain management in elderly patients undergoing gastrectomy, this analgesic strategy had no clear beneficial effects on postoperative complications during the first 30 PODs, morbidity, mortality, or cancer recurrence during the first 2 years after the operation.

\section{REFERENCES}

1. Bentrem DJ, Cohen ME, Hynes DM, Ko CY, Bilimoria KY. Identification of specific quality improvement opportunities for the elderly undergoing gastrointestinal surgery. Arch Surg 2009; 144: 1013-20.

2. Park WY, Thompson JS, Lee KK. Effect of epidural anesthesia and analgesia on perioperative outcome: a randomized, controlled Veterans Affairs cooperative study. Ann Surg 2001; 234: 560-9.

3. Rodgers A, Walker N, Schug S, McKee A, Kehlet H, van Zundert A, et al. Reduction of postoperative mortality and morbidity with epidural or spinal anaesthesia: results from overview of ran- 
domised trials. BMJ 2000; 321: 1493.

4. Gottschalk A, Ford JG, Regelin CC, You J, Mascha EJ, Sessler DI, et al. Association between epidural analgesia and cancer recurrence after colorectal cancer surgery. Anesthesiology 2010; 113: 27-34.

5. Cata JP, Chavez-MacGregor M, Valero V, Black W, Black DM, Goravanchi F, et al. Impact of paravertebral block analgesia on breast cancer survival after surgery. Reg Anesth Pain Med 2016; 41: 696-703.

6. Rigg JR, Jamrozik K, Myles PS, Silbert BS, Peyton PJ, Parsons RW, et al. Epidural anaesthesia and analgesia and outcome of major surgery: a randomised trial. Lancet 2002; 359: 1276-82.

7. American Thoracic Society; Infectious Diseases Society of America. Guidelines for the management of adults with hospital-acquired, ventilator-associated, and healthcare-associated pneumonia. Am J Respir Crit Care Med 2005; 171: 388-416.

8. Huang DD, Zhuang CL, Wang SL, Pang WY, Lou N, Zhou CJ, et al. Prediction of prolonged postoperative ileus after radical gastrectomy for gastric cancer: a scoring system obtained from a prospective study. Medicine (Baltimore) 2015; 94: e2242.

9. Marret E, Remy C, Bonnet F. Meta-analysis of epidural analgesia versus parenteral opioid analgesia after colorectal surgery. Br J Surg 2007; 94: 665-73.

10. Liu SS, Wu CL. Effect of postoperative analgesia on major postoperative complications: a systematic update of the evidence. Anesth Analg 2007; 104: 689-702.

11. Shin HS, Oh SJ, Suh BJ. Factors related to morbidity in elderly gastric cancer patients undergoing gastrectomies. J Gastric Cancer 2014; 14: 173-9.

12. Jørgensen H, Wetterslev J, Møiniche S, Dahl JB. Epidural local anaesthetics versus opioid-based analgesic regimens on postoperative gastrointestinal paralysis, PONV and pain after abdominal surgery. Cochrane Database Syst Rev 2000; (4): CD001893.

13. Kooij FO, Schlack WS, Preckel B, Hollmann MW. Does regional analgesia for major surgery improve outcome? Focus on epidural analgesia. Anesth Analg 2014; 119: 740-4.

14. Kampe S, Weinreich G, Darr C, Eicker K, Stamatis G, Hachenberg $\mathrm{T}$. The impact of epidural analgesia compared to systemic opioid-based analgesia with regard to length of hospital stay and recovery of bowel function: retrospective evaluation of 1555 patients undergoing thoracotomy. J Cardiothorac Surg 2014; 9: 175.

15. Gibson SJ, Helme RD. Age-related differences in pain perception and report. Clin Geriatr Med 2001; 17: 433-56.

16. Huang HW, Wang WC, Lin CC. Influence of age on thermal thresholds, thermal pain thresholds, and reaction time. J Clin Neurosci 2010; 17: 722-6.
17. Revie EJ, Massie LJ, McNally SJ, McKeown DW, Garden OJ, Wigmore SJ. Effectiveness of epidural analgesia following open liver resection. HPB (Oxford) 2011; 13: 206-11.

18. Kim SH, Shin YS, Oh YJ, Park IH, Choi YS. Postoperative patient controlled analgesia in elderly Koreans: epidural versus intravenous administration. Korean J Anesthesiol 2013; 65: 365-7.

19. Nam K, Seo JH, Bahk JH. Unsuspected intravascular migration of a thoracic epidural catheter in a thoracotomy patient: A case report. Anesth Pain Med 2013; 8: 184-6.

20. Ready LB. Acute pain: lessons learned from 25,000 patients. Reg Anesth Pain Med 1999; 24: 499-505.

21. McLeod G, Davies H, Munnoch N, Bannister J, MacRae W. Postoperative pain relief using thoracic epidural analgesia: outstanding success and disappointing failures. Anaesthesia 2001; 56: 75-81.

22. Kamiński JP, Pai A, Ailabouni L, Park JJ, Marecik SJ, Prasad LM, et al. Role of epidural and patient-controlled analgesia in site-specific laparoscopic colorectal surgery. JSLS 2014; 18: e2014.00207.

23. Loran DB, Hyde BR, Zwischenberger JB. Perioperative management of special populations: the geriatric patient. Surg Clin North Am 2005; 85: 1259-66.

24. Brown CH 4th, LaFlam A, Max L, Wyrobek J, Neufeld KJ, Kebaish $\mathrm{KM}$, et al. Delirium after spine surgery in older adults: incidence, risk factors, and outcomes. J Am Geriatr Soc 2016; 64: 2101-8.

25. Exadaktylos AK, Buggy DJ, Moriarty DC, Mascha E, Sessler DI. Can anesthetic technique for primary breast cancer surgery affect recurrence or metastasis? Anesthesiology 2006; 105: 660-4.

26. Cummings KC 3rd, Xu F, Cummings LC, Cooper GS. A comparison of epidural analgesia and traditional pain management effects on survival and cancer recurrence after colectomy: a population-based study. Anesthesiology 2012; 116: 797-806.

27. Myles PS, Peyton P, Silbert B, Hunt J, Rigg JR, Sessler DI. Perioperative epidural analgesia for major abdominal surgery for cancer and recurrence-free survival: randomised trial. BMJ 2011; 342: d1491.

28. Sadurní M, Beltrán de Heredia S, Dürsteler C, Pérez-Ramos A, Langohr K, Escolano F, et al. Epidural vs. intravenous fentanyl during colorectal surgery using a double-blind, double-dummy design. Acta Anaesthesiol Scand 2013; 57: 1103-10.

29. Privado MS, Issy AM, Lanchote VL, Garcia JB, Sakata RK. Epidural versus intravenous fentanyl for postoperative analgesia following orthopedic surgery: randomized controlled trial. Sao Paulo Med J 2010; 128: 5-9.

30. Christopherson R, James KE, Tableman M, Marshall P, Johnson FE. Long-term survival after colon cancer surgery: a variation associated with choice of anesthesia. Anesth Analg 2008; 107: 325-32. 